\title{
MAGNETOELASTIC PLANE WAVES IN ROTATING MEDIA IN THERMOELASTICITY OF TYPE II (G-N MODEL)
}

\section{S. K. ROYCHOUDHURI and MANIDIPA BANERJEE (CHATTOPADHYAY)}

\author{
Received 4 April 2004
}

\begin{abstract}
A study is made of the propagation of time-harmonic plane waves in an infinite, conducting, thermoelastic solid permeated by a uniform primary external magnetic field when the entire medium is rotating with a uniform angular velocity. The thermoelasticity theory of type II (G-N model) (1993) is used to study the propagation of waves. A more general dispersion equation is derived to determine the effects of rotation, thermal parameters, characteristic of the medium, and the external magnetic field. If the primary magnetic field has a transverse component, it is observed that the longitudinal and transverse motions are linked together. For low frequency $(x \ll 1, x$ being the ratio of the wave frequency to some standard frequency $\omega^{*}$ ), the rotation and the thermal field have no effect on the phase velocity to the first order of $x$ and then this corresponds to only one slow wave influenced by the electromagnetic field only. But to the second order of $\chi$, the phase velocity, attenuation coefficient, and the specific energy loss are affected by rotation and depend on the thermal parameters $c_{T}, c_{T}$ being the nondimensional thermal wave speed of G-N theory, and the thermoelastic coupling $\epsilon_{T}$, the electromagnetic parameters $\epsilon_{H}$, and the transverse magnetic field $R_{H}$. Also for large frequency, rotation and thermal field have no effect on the phase velocity, which is independent of primary magnetic field to the first order of $(1 / \chi)(\chi \gg 1)$, and the specific energy loss is a constant, independent of any field parameter. However, to the second order of $(1 / \chi)$, rotation does exert influence on both the phase velocity and the attenuation factor, and the specific energy loss is affected by rotation and depends on the thermal parameters $c_{T}$ and $\epsilon_{T}$, electromagnetic parameter $\epsilon_{H}$, and the transverse magnetic field $R_{H}$, whereas the specific energy loss is independent of any field parameters to the first order of $(1 / \chi)$.
\end{abstract}

2000 Mathematics Subject Classification: 74F05.

1. Introduction. The study of propagation of thermoelastic and magnetothermoelastic waves in a nonrotating medium was made by several authors. Biot [2] derived the equations of thermoelasticity based on the Fourier's law, which is concerned with the interaction of the thermal field and elastic deformation such that these two fields are linked together. Biot's equations have been used for the investigation of the plane thermoelastic waves. The main drawback of Biot's equations is that they were based on the Fourier's law which predicts an infinite speed of propagation of heat. Lord and Shulman [11] employed a modified version of the Fourier law to eliminate this paradox and thereby established the generalized coupled heat conduction equation which is hyperbolic in nature. They have derived equations of dynamic thermoelasticity based on the modified Fourier's law and these equations are usually regarded as the basis of generalized thermoelasticity. Lord and Shulman's equations have been used by 
several authors including Puri [16], Nayfeh and Nemat-Nasser [12] to study the plane thermoelastic waves in an unbounded isotropic homogeneous elastic medium. Agarwal [1] investigated the propagation of surface waves in generalized thermoelasticity.

Paria [14] and Wilson [24] investigated the propagation of magnetothermoelastic waves in a nonrotating medium. These studies, based on the theory of classical coupled thermoelasticity, were essentially concerned with the interaction of the electromagnetic field, the thermal field, and the elastic field as well as the dispersion relation. The propagation of harmonic plane waves in a rotating elastic medium has been investigated by Schoenberg and Censor [22] in some details. It has been shown that the rotation causes the elastic medium to be dispersive and anisotropic. This study included some discussion on the free surface phenomenon in a rotating half-space. Results concerning slowness surfaces, energy flux, reflected waves, and generalized Rayleigh waves have been obtained.

It seems relevant from the above discussion that little attention has been given to the study of propagation of thermoelastic plane waves in a rotating medium in the presence of external magnetic field based on the generalized thermoelasticity. In view of the fact that most large bodies, like the earth, the moon, and other planets, have an angular velocity, it is important to consider the propagation of magnetothermoelastic plane waves in an electrically conducting, rotating elastic medium under the action of the external magnetic field with or without thermal relaxation. In this connection, Roychoudhuri and Debnath $[17,18,19,21,20]$ have studied propagation of magnetothermoelastic plane waves in a rotating thermoelastic medium permeated by a primary uniform magnetic field by using the generalized heat conduction equation of Lord and Shulman. In the present problem, we have studied the propagation of time-harmonic coupled electromagnetoelastic dilatational thermal shear waves using the thermoelasticity theory of type II [9] (Green-Naghdi (G-N) model 1993). This thermoelastic model possesses several significant characteristics that differ from the traditional classical development in thermoelastic material behaviors: (i) it does not sustain energy dissipation, (ii) the entropy flux vector (or equivalently heat flow vector) in the theory is determined in terms of the same potential that also determines the stress, (iii) it permits transmission of heat flow as thermal waves at finite speed. Several problems in thermoelasticity relating to this Green-Naghdi theory of thermoelasticity of type II (without thermal energy dissipation) have been studied by several authors $[4,5,6,7,8,23]$. In this paper, G-N model of thermoelasticity of type II is used to obtain a more general dispersion equation to ascertain the effects of rotation, thermal parameter $c_{T}$, the nondimensional thermal wave speed characteristic of G-N theory, thermoelastic coupling constant $\epsilon_{T}$ and the external magnetic field on the phase velocity and attenuation factor of the coupled electromagneto dilatational thermal shear waves. Special attention is given to study the effects on the specific energy loss for both low and high frequencies. Though several problems of coupled wave propagation have been studied in generalized magnetothermoelasticity with/without thermal relaxation by previous researchers, it is believed that this particular problem of coupled wave propagation in a rotating magnetoelastic solid using the theory of thermoelasticity of type II (G-N model) [9] has not been dealt with before. 
2. Formulation of the problem and the basic equations. We consider an infinite homogenous, isotropic, thermally, and electrically conducting elastic solid permeated by a primary uniform magnetic field $\vec{B}_{0}=\left(B_{1}, B_{2}, B_{3}\right)$. The elastic medium is characterized by the density $\rho$ and Lamé constants $\lambda, \mu$ and is uniformly rotating with an angular velocity $\vec{\Omega}=\Omega \vec{n}$, where $\vec{n}$ is the unit vector representing the direction of the axis of rotation. The displacement equation of motion in the rotating frame of reference has two additional terms - the centripetal acceleration $\vec{\Omega} \times(\vec{\Omega} \times \vec{u})$ due to the time-varying motion only and the Coriolis acceleration $2 \cdot \vec{\Omega} \times \dot{\vec{u}}$, where $\vec{u}$ is the dynamic displacement vector. These terms do not appear in the nonrotating medium. The dynamic displacement vector is actually measured from a steady-state deformed position and the deformation is assumed to be small. The displacement equations of motion with the increase of temperature $\theta$ above the reference temperature $T_{0}$ is

$$
\mu \nabla^{2} \vec{u}+(\lambda+\mu) \vec{\nabla}(\vec{\nabla} \cdot \vec{u})+\vec{J} \times \vec{B}-v \vec{\nabla} \theta=\rho[\ddot{\vec{u}}+\vec{\Omega} \times(\vec{\Omega} \times \vec{u})+2 \vec{\Omega} \times \dot{\vec{u}}],
$$

where $\vec{J} \times \vec{B}$ is the electromagnetic body force, $\vec{J}$ is the current density, $\vec{B}=\vec{B}_{0}+\vec{b}$ is the total magnetic field, $\vec{b}=\left(b_{x}, b_{y}, b_{z}\right)$ is the perturbed magnetic field which is assumed to be small so that the products with $\vec{b}$ and $\vec{u}$ and their derivatives can be neglected for linearization of the field equations, $v=(3 \lambda+2 \mu) \alpha_{t}, \alpha_{t}$ is the coefficient of linear thermal expansion of the solid, and dots represent the derivatives with respect to time $t$.

The coupled heat conduction equation of the theory of thermoelasticity (type II) without energy dissipation proposed by Green and Naghdi [9] is

$$
\rho C_{\nu} \ddot{\vec{\theta}}+v T_{0} \ddot{\vec{\Delta}}=\rho Q+k^{*} \nabla^{2} \theta
$$

where $C_{v}$ is the specific heat of the solid at constant volume, $\rho$ is the density of the medium, $T_{0}$ is the initial reference temperature, $k^{*}$ is a material constant characteristic of the theory, $Q$ is the heat source function, and $\Delta$ is the dilatation so that $\Delta=\operatorname{div} \vec{u}$. The finite thermal wave speed is $\left(k^{*} / \rho C_{v}\right)^{1 / 2}$.

In the present problem, $Q=0$, so that the heat conduction equation becomes

$$
\rho C_{v} \ddot{\theta}+v T_{0} \ddot{\Delta}=k^{*} \nabla^{2} \theta \text {. }
$$

Equation (2.3) permits thermal wave propagation without damping. The equations (2.1) and (2.3) are to be supplemented by generalized Ohm's law in a continuous medium with Maxwell's electromagnetic field equations.

The electromagnetic field is governed by the Maxwell's equations with the displacement current and charge density neglected as

$$
\begin{aligned}
& \vec{\nabla} \times \vec{H}=\vec{J}, \\
& \vec{\nabla} \times \vec{E}=-\frac{\partial B}{\partial t}, \\
& \vec{\nabla} \cdot \vec{B}=0,
\end{aligned}
$$

where $\vec{B}=\mu_{e} \vec{H}$ and $\mu_{e}$ is the magnetic permeability. 
The generalized Ohm's law is

$$
\vec{J}=\sigma\left[\vec{E}+\left(\frac{\partial \vec{u}}{\partial t}+\vec{\Omega} \times \vec{u}\right) \times \vec{B}\right]
$$

where the time-independent part of $\vec{\Omega} \times \vec{u}$ is neglected, $\sigma$ is the electrical conductivity, $\partial \vec{u} / \partial t$ is the particle velocity of the medium, and the small effect of temperature gradient on $\vec{J}$ is also ignored.

3. Plane wave solutions and dispersion relation. We consider the propagation of plane waves in the rotating medium in the $x$-direction so that all quantities are proportional to $\exp [i(k x-\omega t)]$, where $(\omega / 2 \pi)$ is the wave frequency and $(2 \pi / k)$ is the wave length. We will assume that $\omega$ is real, but $k$ may be complex. The analysis will be carried out without any discussion of the time-independent stresses and displacements that are caused by the centrifugal force and other possible body forces. We look for timevarying dynamic solutions and as such, the time-independent part of the centripetal acceleration as well as all body forces will be neglected. However, the time-dependent part of the electromagnetic body force will be taken into consideration. In view of the above assumptions, we write all field quantities in the form

$$
\begin{gathered}
\vec{u}=(p, q, r)=\left(p_{0}, q_{0}, r_{0}\right) \exp [i(k x-\omega t)], \\
\theta=\theta_{0} \exp [i(k x-\omega t)], \\
\vec{J}=\left(j_{1}, j_{2}, j_{3}\right) \exp [i(k x-\omega t)], \\
\vec{b}=\left(b_{x}, b_{y}, b_{z}\right)=\left(b_{1}, b_{2}, b_{3}\right) \exp [i(k x-\omega t)], \\
\vec{E}=\left(E_{x}, E_{y}, E_{z}\right), \\
\vec{\Omega}=\left(\Omega_{1}, \Omega_{2}, \Omega_{3}\right),
\end{gathered}
$$

where $p_{0}, q_{0}, r_{0} ; j_{1}, j_{2}, j_{3} ; b_{1}, b_{2}, b_{3} ; \Omega_{1}, \Omega_{2}, \Omega_{3}$, and $\theta_{0}$ are all constants.

It follows from (2.4c) that $\operatorname{div} \vec{b}=0$ which implies $b_{x}=0$, since initially $\vec{b}=\overrightarrow{0}$. Also, it follows from (2.4a) that $\mu_{e} \vec{J}=\vec{\nabla} \times \vec{b}$ so that

$$
\begin{gathered}
\vec{J}=\left[0,-\frac{i k}{\mu_{e}} b_{z}, \frac{i k}{\mu_{e}} b_{y}\right], \\
\vec{J} \times \vec{B}_{0}=\left[-\frac{i k}{\mu_{e}}\left(b_{z} B_{3}+b_{y} B_{2}\right), \frac{i k}{\mu_{e}} b_{y} B_{1}, \frac{i k}{\mu_{e}} b_{z} B_{1}\right] .
\end{gathered}
$$

Thus, the term $\vec{J} \times \vec{B}$ in (2.1) can be replaced by $\vec{J} \times \vec{B}_{0}$ given by (3.6).

Substituting (3.1) and (3.2a) into (2.3), we find

$$
\begin{gathered}
\theta_{0}=\alpha p_{0}, \\
\alpha=\frac{i v T_{0} k \omega^{2}}{k^{*} k^{2}-\rho C_{v} \omega^{2}} .
\end{gathered}
$$

Again, $\vec{\nabla} \times \vec{E}=-\partial \vec{b} / \partial t$ gives

$$
\vec{E}=\left(E_{x}, E_{y}, E_{z}\right)=\left(E_{x}, \frac{\omega}{k} b_{z},-\frac{\omega}{k} b_{y}\right) .
$$


Replacing $\vec{B}$ by primary magnetic field $\vec{B}_{0}$, (2.5) takes the form

$$
\vec{J}=\sigma\left[\vec{E}+\left(\frac{\partial u}{\partial t}+\vec{\Omega} \times \vec{u}\right) \times \vec{B}_{0}\right] .
$$

Making use of (3.1) and (3.8) and neglecting the product terms, equation (3.9) with $\vec{J}=\left(J_{x}, J_{y}, J_{z}\right)$ yields

$$
\begin{aligned}
& J_{x}=\sigma\left[E_{x}-i \omega\left(q B_{3}-r B_{2}\right)+B_{3}\left(p \Omega_{3}-r \Omega_{1}\right)-B_{2}\left(q \Omega_{1}-p \Omega_{2}\right)\right], \\
& J_{y}=\sigma\left[\frac{\omega}{k} b_{z}-i \omega\left(r B_{1}-p B_{3}\right)+B_{1}\left(q \Omega_{1}-p \Omega_{2}\right)-B_{3}\left(r \Omega_{2}-q \Omega_{3}\right)\right], \\
& J_{z}=\sigma\left[-\frac{\omega}{k} b_{y}-i \omega\left(p B_{2}-B_{1} q\right)+B_{2}\left(r \Omega_{2}-q \Omega_{3}\right)-B_{1}\left(p \Omega_{3}-r \Omega_{1}\right)\right] .
\end{aligned}
$$

Elimination of $\vec{J}$ from (3.5) and (3.10), (3.11), and (3.12) gives

$$
\begin{gathered}
\sigma\left[E_{x}-i \omega\left(q B_{3}-r B_{2}\right)+B_{3}\left(p \Omega_{3}-r \Omega_{1}\right)-B_{2}\left(q \Omega_{1}-p \Omega_{2}\right)\right]=0, \\
\sigma\left[\frac{\omega}{k} b_{z}-i \omega\left(r B_{1}-p B_{3}\right)+B_{1}\left(q \Omega_{1}-p \Omega_{2}\right)-B_{3}\left(r \Omega_{2}-q \Omega_{3}\right)\right]=-\frac{i k}{\mu_{e}} b_{z}, \\
\sigma\left[-\frac{\omega}{k} b_{y}-i \omega\left(p B_{2}-q B_{1}\right)+B_{2}\left(r \Omega_{2}-q \Omega_{3}\right)-B_{1}\left(p \Omega_{3}-r \Omega_{1}\right)\right]=\frac{i k}{\mu_{e}} b_{y} .
\end{gathered}
$$

From (3.13) we get

$$
E_{x}=i \omega\left(q B_{3}-r B_{2}\right)-B_{3}\left(p \Omega_{3}-r \Omega_{1}\right)+B_{2}\left(q \Omega_{1}-p \Omega_{2}\right) .
$$

We next put (3.1)-(3.4) into (2.1) and suppress the factor exp[i(kx-wt)] throughout the subsequent discussion to obtain the following equations:

$$
\begin{gathered}
p_{0}\left[\rho\left(\Omega_{1}^{2}-\Omega^{2}-\omega^{2}\right)+(\lambda+2 \mu) k^{2}+i v \alpha k\right]+q_{0}\left[\rho\left(2 i \omega \Omega_{3}+\Omega_{1} \Omega_{2}\right)\right] \\
+r_{0}\left[\rho\left(\Omega_{1} \Omega_{3}-2 i \omega \Omega_{2}\right)\right]+\frac{i k}{\mu_{e}}\left(b_{3} B_{3}+b_{2} B_{2}\right)=0, \\
p_{0}\left[\rho\left(\Omega_{1} \Omega_{2}-2 i \omega \Omega_{3}\right)\right]+q_{0}\left[\rho\left(\Omega_{2}^{2}-\Omega^{2}-\omega^{2}\right)+\mu k^{2}\right]+r_{0}\left[\rho\left(\Omega_{2} \Omega_{3}+2 i \omega \Omega_{1}\right)\right]-\frac{i k}{\mu_{e}} b_{2} B_{1}=0, \\
p_{0}\left[\rho\left(\Omega_{1} \Omega_{3}+2 i \omega \Omega_{2}\right)\right]+q_{0}\left[\rho\left(\Omega_{2} \Omega_{3}-2 i \omega \Omega_{1}\right)\right]+r_{0}\left[\rho\left(\Omega_{3}^{2}-\Omega^{2}-\omega^{2}\right)+\mu k^{2}\right]-\frac{i k}{\mu_{e}} b_{3} B_{1}=0, \\
p_{0}\left[\sigma\left(i \omega B_{3}-B_{1} \Omega_{2}\right)\right]+q_{0}\left[\sigma\left(B_{1} \Omega_{1}+B_{3} \Omega_{3}\right)\right]+r_{0}\left[-\sigma\left(i \omega B_{1}+B_{3} \Omega_{2}\right)\right]+b_{3}\left[\frac{i k}{\mu_{e}}+\frac{\sigma \omega}{k}\right]=0, \\
\left.p_{0}\left[-\sigma\left(i \omega B_{2}+B_{1} \Omega_{3}\right)\right]+q_{0}\left[\sigma\left(i \omega B_{1}-B_{2} \Omega_{3}\right)\right]+r_{0}\left[\sigma\left(B_{2} \Omega_{2}+B_{1} \Omega_{1}\right)\right]-b_{2}\left[\frac{i k}{\mu_{e}}+\frac{\sigma \omega}{k}\right]=0.20\right)
\end{gathered}
$$

Equations (3.17)-(3.21) constitute a system of five equations with five unknowns, $p_{0}$, $q_{0}, r_{0}$ and the perturbed quantities $b_{2}, b_{3}$. 
Since $\vec{b}=(0, b y, b z)$ and $\vec{b}$-field is normal to the $x$-axis, we then choose the $y$-axis and the $z$-axis such that $\vec{b}$-field is along the $y$-axis. Invoking the additional assumption $\Omega_{1}=\Omega_{2}=0$ and $\Omega_{3}=\Omega \neq 0$ and considering that $r_{0} \equiv 0$ provided $\mu k^{2}-\rho \omega^{2} \neq 0$ (evident from (3.19)) so that $B_{3} \equiv 0$, we set the applied and perturbed magnetic fields to be $\left(B_{1}, B_{2}, 0\right)$ and $\left(0, b_{2}, 0\right)$, respectively.

This leads to the following three homogenous equations with three unknowns $p_{0}$, $q_{0}$, and $b_{2}$

$$
\begin{gathered}
p_{0}\left[-\rho\left(\Omega^{2}+\omega^{2}\right)+(\lambda+2 \mu) k^{2}+i v \alpha k\right]+2 i \omega \rho \Omega q_{0}+\frac{i k}{\mu_{e}} B_{2} b_{2}=0, \\
p_{0}[-2 i \omega \rho \Omega]+q_{0}\left[\mu k^{2}-\rho\left(\Omega^{2}+\omega^{2}\right)\right]-\frac{i k B_{1}}{\mu_{e}} b_{2}=0, \\
p_{0}\left[-\sigma\left(B_{1} \Omega+i \omega B_{2}\right)\right]+q_{0}\left[\sigma\left(i \omega B_{1}-\Omega B_{2}\right)\right]-\left[\frac{i k}{\mu_{e}}+\frac{\sigma \omega}{k}\right] b_{2}=0 .
\end{gathered}
$$

Elimination of $p_{0}, q_{0}$, and $b_{2}$ gives the dispersion equation

$$
\left|\begin{array}{ccc}
-\rho\left(\Omega^{2}+\omega^{2}\right)+(\lambda+2 \mu) k^{2}+i v \alpha k & 2 i \omega \rho \Omega & \frac{i k B_{2}}{\mu_{e}} \\
-2 i \omega \rho \Omega & \mu k^{2}-\rho\left(\Omega^{2}+\omega^{2}\right) & -\frac{i k B_{1}}{\mu_{e}} \\
\sigma\left(B_{1} \Omega+i \omega B_{2}\right) & \sigma\left(\Omega B_{2}-i \omega B_{1}\right) & \left(\frac{i k}{\mu_{e}}+\frac{\sigma \omega}{k}\right)
\end{array}\right|=0 .
$$

It follows from the dispersion equation that the significant effects of the rotation and the thermal field on the phase velocity $\omega / \operatorname{Re}(k)$ are reflected through the terms involving $\Omega$ and the term containing $\alpha$ through the term $k^{*}$, characteristic of G-N theory.

In order to make further simplification of the dispersion equation, we assume $\vec{B}_{0}=$ $\left(0, B_{2}, 0\right)$ so that $(3.25)$ becomes

$$
\left|\begin{array}{ccc}
-\rho\left(\omega^{2}+\Omega^{2}\right)+(\lambda+2 \mu) k^{2}+i v \alpha k & 2 i \omega \rho \Omega & \frac{i k}{\mu_{e}} B_{2} \\
-2 i \omega \rho \Omega & \mu k^{2}-\rho\left(\Omega^{2}+\omega^{2}\right) & 0 \\
\sigma i \omega B_{2} & \sigma \Omega B_{2} & \frac{i k}{\mu_{e}}+\frac{\sigma \omega}{k}
\end{array}\right|=0 .
$$

Expanding this determinant and substituting $(\Omega / \omega)=\Omega_{0}$ and the value of $\alpha$ from (3.7b), we obtain

$$
\begin{aligned}
& {\left[\left\{-\rho \omega^{2}\left(1+\Omega_{0}^{2}\right)+(\lambda+2 \mu) k^{2}\right\} \cdot\left(k^{*} k^{2}-\rho C_{v} \omega^{2}\right)-v^{2} k^{2} T_{0} \omega^{2}\right]} \\
& \times\left[-\rho \omega^{2}\left(1+\Omega_{0}^{2}\right)+\mu k^{2}\right]\left(\frac{i k}{\mu_{e}}+\frac{\sigma \omega}{k}\right)+\left(k^{*} k^{2}-\rho C_{v} \omega^{2}\right) \\
& \times\left[-4 \omega^{2} \rho^{2} \Omega^{2}\left(\frac{i k}{\mu_{e}}+\frac{\sigma \omega}{k}\right)+\frac{k \sigma \omega B_{2}^{2}}{\mu_{e}}\left\{2 \rho \Omega^{2}-\rho \omega^{2}\left(1+\Omega_{0}^{2}\right)+\mu k^{2}\right\}\right]=0 .
\end{aligned}
$$


It is convenient to introduce the following dimensionless quantities:

$$
\begin{gathered}
x=\frac{\omega}{\omega^{*}}, \quad \xi=\frac{k c_{1}}{\omega^{*}}, \quad \epsilon_{T}=\frac{T_{0} v^{2}}{\rho^{2} C_{v} c_{1}^{2}}, \quad \epsilon_{H}=\frac{\omega^{*} v_{H}}{c_{1}^{2}}, \quad \gamma_{H}=\left(\mu_{e} \cdot \sigma\right)^{-1}, \\
\frac{k^{*}}{\rho C_{v} c_{1}^{2}}=\frac{k^{*} / \rho C_{v}}{c_{1}^{2}}=c_{T}^{2},
\end{gathered}
$$

where $c_{1}=((\lambda+2 \mu) / \rho)^{1 / 2}$ is the longitudinal elastic wave velocity, $\epsilon_{T}$ is the thermoelastic coupling constant, $c_{T}$ is the nondimensional thermal wave speed of G-N theory, depending on $k^{*}, \omega^{*}$ is some standard frequency, $v_{H}$ is the magnetic viscosity.

We divide (3.27) by $c_{1}^{2}$ and observe the following results for further simplification of (3.27):

$$
\begin{gathered}
-\frac{\omega^{2}}{c_{1}^{2}}\left(\Omega_{0}^{2}+1\right)+k^{2}=\frac{\omega^{* 2}}{c_{1}^{2}}\left\{\xi^{2}-\chi^{2}\left(1+\Omega_{0}^{2}\right)\right\}, \\
\kappa^{*} k^{2}-\rho C_{\nu} \omega^{2}=-k^{*} \frac{\omega^{* 2}}{c_{1}^{2}}\left(\xi^{2}-\frac{\chi^{2}}{c_{T}^{2}}\right), \\
\frac{v^{2} k^{2} T_{0} \omega^{2}}{\rho c_{1}^{2}}=\frac{\epsilon_{T}}{c_{T}^{2}} \xi^{2} \chi^{2} \frac{k^{*} \omega^{* 4}}{c_{1}^{4}}, \\
\mu k^{2}-\rho \omega^{2}\left(\Omega_{0}^{2}+1\right)=\rho \omega^{* 2}\left\{s^{2} \xi^{2}-\chi^{2}\left(\Omega_{0}^{2}+1\right)\right\}, \\
\frac{k^{2}}{\sigma \mu_{e}}-i \omega=-i \omega^{*}\left(x+i \xi^{2} \epsilon_{H}\right), \\
2 \rho \Omega^{2}-\rho\left(\Omega_{0}^{2}+1\right) \omega^{2}+\mu k^{2}=\rho \omega^{* 2}\left\{\chi^{2}\left(\Omega_{0}^{2}-1\right)+s^{2} \xi^{2}\right\},
\end{gathered}
$$

where $s^{2}=\left(c_{2} / c_{1}\right)^{2}$ and $c_{2}^{2}=\mu / \rho$.

Introducing the magnetic pressure number $R_{H}=B_{2}^{2} / \rho c_{1}^{2} \mu_{e}$ as defined by Pai [13], (3.27) takes the form

$$
\begin{aligned}
& {\left[\left\{\xi^{2}-\chi^{2}\left(\Omega_{0}^{2}+1\right)\right\}\left(\xi^{2} c_{T}^{2}-\chi^{2}\right)-\epsilon_{T} \xi^{2} \chi^{2}\right]\left\{s^{2} \xi^{2}-\chi^{2}\left(\Omega_{0}^{2}+1\right)\right\}\left(\chi+i \xi^{2} \epsilon_{H}\right)} \\
& +\left(\xi^{2} c_{T}^{2}-\chi^{2}\right)\left[-4 \Omega_{0}^{2} \chi^{4}\left(\chi+i \xi^{2} \epsilon_{H}\right)+R_{H} \xi^{2} \chi\left\{\chi^{2}\left(\Omega_{0}^{2}-1\right)+s^{2} \xi^{2}\right\}\right]=0 .
\end{aligned}
$$

The equation indicates the influence of the rotation and the thermal field through $c_{T}$ and $\epsilon_{T}$ on the phase velocity. In the absence of rotation $\left(\Omega_{0}=0\right)$, the dispersion relation (3.35) reduces to

$$
\begin{aligned}
\left(s^{2} \xi^{2}-\chi^{2}\right)[ & \left(\xi^{2} c_{T}^{2}-\chi^{2}\right)\left(\xi^{2}-\chi^{2}\right)\left(\chi+i \xi^{2} \epsilon_{H}\right) \\
& \left.-\chi^{2} \xi^{2} \epsilon_{T}\left(\chi+i \xi^{2} \epsilon_{H}\right)+R_{H} \chi \xi^{2}\left(\xi^{2} c_{T}^{2}-\chi^{2}\right)\right]=0 .
\end{aligned}
$$

In this case, the phase velocity is broken up into two factors. The first factor corresponds to $s^{2} \xi^{2}-\chi^{2}=0$ which leads to a transverse elastic wave.

The other factor leads to

$$
\left[\left(\xi^{2}-\chi^{2}\right)\left(\chi+i \xi^{2} \epsilon_{H}\right)+R_{H} \chi \xi^{2}\right]\left(\xi^{2} c_{T}^{2}-\chi^{2}\right)-\epsilon_{T} \chi^{2} \xi^{2}\left(\chi+i \xi^{2} \epsilon_{H}\right)=0 .
$$


Equation (3.37) corresponds to the dispersion equation of coupled magnetoelastic dilatational thermal waves influenced by $k^{*}$ through $c_{T}$, a characteristic of the material of G-N model and is not so far dealt with.

Setting $R_{H}=0$, the dispersion equation (3.37) reduces to

$$
\left(\chi+i \xi^{2} \epsilon_{H}\right)\left[\left(\xi^{2}-\chi^{2}\right)\left(\xi^{2} c_{T}^{2}-\chi^{2}\right)-\epsilon_{T} \chi^{2} \xi^{2}\right]=0 .
$$

The first factor corresponds to quasistatic oscillations of the electromagnetic field, not coupled with the displacement field (Parkus [15]). The second factor of (3.38) corresponds to dispersion equation (not considered so far) for purely thermoelastic waves (G-N model) leading to $\left(\xi^{2}-\chi^{2}\right)\left(\xi^{2} c_{T}^{2}-\chi^{2}\right)-\epsilon_{T} \chi^{2} \xi^{2}=0$ in contrast to the equation derived by Chadwick [3] in classical coupled thermoelastic theory. The roots of this equation are real, indicating that purely thermoelastic waves in thermoelasticity of type II (G-N model) are unattenuated and nondispersive (without energy dissipation), not yet considered.

The roots of the dispersion equation for purely thermoelastic waves in thermoelasticity of type II (G-N model) are $\xi^{2}=\left(M_{1} \pm N_{1}\right) \chi^{2}$, where $M_{1}=\left(1 / 2 c_{T}^{2}\right)\left(c_{T}^{2}+1+\epsilon_{T}\right)$ and $N_{1}=\left(1 / 2 c_{T}^{2}\right)\left[\left(c_{T}^{2}-1\right)^{2}+\epsilon_{T}^{2}+2 \epsilon_{T}\left(c_{T}^{2}+1\right)\right]^{1 / 2}$ (imposing the condition that $c_{T}>1$ ) and the phase speeds are $C_{p}^{E, T}=\chi c_{1} / \xi=c_{1} / \sqrt{\left(M_{1} \pm N_{1}\right)}=V_{E}, V_{T}$ corresponding to + ve and - ve signs.

Setting $\epsilon_{T}=0$ leads to $V_{E}=c_{1}$, which is the elastic dilatational wave speed and $V_{T}=\sqrt{k^{*} / \rho C_{v}}=$ finite thermal wave speed of G-N model. Thus, $V_{E}$ corresponds to modified elastic dilatational wave speed and $V_{T}$ the modified thermal wave speed, modified by the nondimensional thermal wave speed $c_{T}$ of G-N theory and the thermoelastic coupling $\epsilon_{T}$. Clearly, $V_{E}<V_{T}$, implying that modified elastic wave follows the modified thermal wave.

Equation (3.35) represents a more general dispersion relation in the sense that it incorporates the effects of rotation, the finite thermal wave speed $c_{T}$, thermal coupling $\epsilon_{T}$, and $R_{H}$. Also, it shows that if the primary magnetic field has a transverse component, the longitudinal and transverse components of the displacement vector are linked together.

As (3.35) is very complicated, we consider the following limiting cases in order to examine the effects of the rotation, the finite thermal wave speed $c_{T}$, thermal coupling $\epsilon_{T}$, external magnetic field $R_{H}$ on the phase velocity, and attenuation coefficient of waves and on specific energy loss.

4. Low-frequency region $(x \ll 1)$. In this case, the wave frequency $\omega$ is much smaller than the characteristic frequency $\omega^{*}$. We consider this case with finite electrical conductivity $\left(\sigma \neq 0, \nu_{H} \neq 0\right)$. Thus, when $\chi=0, \xi^{2}=0$ so that we can write $\xi^{2}=i \phi \chi+0\left(\chi^{2}\right)$, where $\phi$ is to be determined. We substitute $\xi^{2}$ into (3.35), retain the terms containing $\chi^{4}$ and then equate the coefficient of $\chi^{4}$ to zero in order to obtain an equation for $\phi$ as

$$
\phi=\frac{1+R_{H}}{\epsilon_{H}} .
$$


This corresponds to one kind of slow wave, because

$$
\left|\frac{\omega}{k}\right|=\left|\frac{c_{1} X}{\xi}\right| \sim c_{1} 0\left(\chi^{1 / 2}\right) \ll c_{1} .
$$

Thus, for the low frequency, the rotation and the thermal field have no influence on the phase velocity in the case of finite conductivity. This corresponds to only one slow wave coupled to the electromagnetic field only in contrast to (4.1) derived by Roychoudhuri and Debnath [20]. Then, the phase velocity can be found from the result

$$
\xi= \pm(1+i)\left(\frac{\chi}{2 \epsilon_{H}}\right)^{1 / 2} R_{m}
$$

where $R_{m}^{2}=1+R_{H}=1+v_{A}^{2} / c_{1}^{2}$ and $v_{A}$ is the Alfvén wave velocity.

It follows from (4.3) that there exists a magnetoelastic wave.

It follows from the real and imaginary part of $\xi$ that the phase velocity is

$$
c_{p}=c_{1}\left(\frac{\chi \epsilon_{H}}{2}\right)^{1 / 2} R_{m}^{-1}
$$

The attenuation factor is

$$
a_{f}=\frac{\omega^{*}}{c_{1}}\left(\frac{\chi}{2 \epsilon_{H}}\right)^{1 / 2} R_{m}
$$

The phase speed and attenuation factor are independent of $c_{T}$, the thermal wave speed, and thermoelastic coupling $\epsilon_{T}$ but influenced by $R_{H}$ to the order of $(\chi)$ for $\chi \ll 1$.

However, considering terms of $0\left(\chi^{2}\right)$ for $\chi \ll 1$, we obtain from the general dispersion equation (3.35)

$$
\begin{gathered}
\xi^{2}=i M_{2}+N_{2}, \quad M_{2}=\frac{\left(1+R_{H}\right) \chi}{\epsilon_{H}} \\
N_{2}=\frac{\left\{c_{T}^{2} \cdot s^{2}\left(1+\Omega_{0}^{2}\right)+s^{2}\left(1+\epsilon_{T}\right)+\left(1+\Omega_{0}^{2}\right) c_{T}^{2}\right\} \chi^{2}}{s^{2} c_{T}^{2}} .
\end{gathered}
$$

It follows from the real and imaginary parts of $\xi$ that the phase velocity is

$$
c_{p}=R_{e}\left(\frac{\chi c_{1}}{\xi}\right)=\frac{\chi c_{1}}{\sqrt{R_{1}}} \cos \frac{\phi}{2}
$$

and the attenuation factor is

$$
a_{f}=\frac{\omega^{*}}{c_{1}} \sqrt{R_{1}} \sin \frac{\phi}{2}
$$

where $R_{1}=\left(M_{2}^{2}+N_{2}^{2}\right)^{1 / 2}$ and $\tan \phi=M_{2} / N_{2}$. 
This confirms that the phase speed and the attenuation factors are both affected by rotation, finite thermal wave speed $c_{T}$, the thermoelastic coupling $\epsilon_{T}$, the external magnetic field, and the electromagnetic parameter.

5. High-frequency region $(x \gg 1)$. This case corresponds to the case of wave frequency $\omega$ much larger than $\omega^{*}$. Dividing the dispersion equation (3.35) by $\chi^{7}$ and neglecting all terms involving the second and higher powers of $(1 / \chi)$, we obtain

$$
\xi= \pm(1+i)\left(\frac{\chi}{2 \epsilon_{H}}\right)^{1 / 2}
$$

Thus, no effect of rotation and $c_{T}, \epsilon_{T}$ on the phase velocity is observed to the first-order of $(1 / \chi)$ for $(\chi \gg 1)$. Also, the phase speed does not depend on the primary magnetic field, but it depends on the magnetic and the electrical property of the medium.

To the first order of $(1 / \chi)$ for $(\chi \gg 1)$, the phase velocity $c_{p}$ and the attenuation factor $a_{f}$ are given by

$$
\begin{aligned}
& c_{p}=c_{1}\left(\frac{\epsilon_{H} \cdot \chi}{2}\right)^{1 / 2}, \\
& a_{f}=\frac{\omega^{*}}{c_{1}}\left(\frac{\chi}{2 \epsilon_{H}}\right)^{1 / 2} .
\end{aligned}
$$

Now, dividing (3.35) by $\chi^{7}$ and retaining the terms of the order of $(1 / \chi)^{2}$ for $(\chi \gg 1)$ and neglecting the higher powers of $(1 / \chi)$, we obtain

$$
\xi^{2}=\frac{(L+i M)}{L^{2}+M^{2}}
$$

where

$$
\begin{gathered}
M=\frac{\epsilon_{H}}{\chi}, \\
L=\frac{1}{\chi^{2}\left(\Omega_{0}^{2}-1\right)^{2}}\left[\left(1+\epsilon_{T}+s^{2}\right)\left(\Omega_{0}^{2}+1\right)-R_{H}\left(\Omega_{0}^{2}-1\right)+\left(\Omega_{0}^{2}-1\right)^{2} c_{T}^{2}\right]=\frac{L_{0}}{\chi^{2}},
\end{gathered}
$$

where

$$
L_{0}=\frac{1}{\left(\Omega_{0}^{2}-1\right)^{2}}\left[\left(1+\epsilon_{T}+s^{2}\right)\left(\Omega_{0}^{2}+1\right)^{2}+\left(\Omega_{0}^{2}-1\right) c_{T}^{2}-R_{H}\left(\Omega_{0}^{2}-1\right)\right]
$$

It follows from the real and the imaginary parts of $\xi$ that the phase velocity is

$$
c_{p}=\frac{X c_{1}}{\sqrt{R}} \cos \frac{\theta}{2}
$$

and the attenuation factor is

$$
a_{f}=\frac{\omega^{*}}{c_{1}} \sqrt{R} \sin \frac{\theta}{2},
$$


where

$$
R=\frac{1}{\sqrt{L^{2}+M^{2}}}, \quad \tan \theta=\frac{M}{L} .
$$

The results (5.7) and (5.8) are similar to (5.3) and (5.4) reported by Roychoudhuri and Debnath [20].

It is important to observe that rotation, thermal parameter $k^{*}$ (and hence $c_{T}$ ), characteristic of G-N theory and the thermoelastic coupling constant $\epsilon_{T}$, do exert influence on both the phase velocity and the attenuation factor for high frequencies to the second order of $(1 / \chi)$. Also, both the phase speed and the attenuation factor are modified by the applied magnetic field through the term $L$ for high frequency. This fact was not noticed for the case of low frequency.

6. Specific energy loss. Making reference to Kolsky [10], the specific energy loss $(\Delta W / W)$ is defined as the ratio of the energy dissipated per stress cycle to the total vibrational energy and is given by

$$
\frac{\Delta W}{W}=\frac{4 \pi}{\omega} c_{p} a_{f}
$$

To the second order of $\chi$ for $\chi \ll 1$, the specific energy loss from (4.7) and (4.8) is given by

$$
\frac{\Delta W}{W}=2 \pi \frac{M_{2}}{\left(M_{2}^{2}+N_{2}^{2}\right)^{1 / 2}} .
$$

Therefore, the specific energy loss is affected by rotation, finite thermal wave speed $c_{T}$, the thermoelastic coupling $\epsilon_{T}$, the external magnetic field, and the electromagnetic parameter.

For $\chi \gg 1$, the specific energy loss to the first order of $1 / \chi$ is obtained from (5.2) and (5.3) in the form

$$
\frac{\Delta W}{W}=2 \pi
$$

Equation (6.3) shows that the specific energy loss is independent of any field parameters in the case of high frequency up to the first order of $(1 / \chi)$.

However, to the second order of $(1 / \chi)$, the expression for the specific energy loss is obtained from (5.7) and (5.8) in the form

$$
\frac{\Delta W}{W}=2 \pi \frac{M}{\sqrt{L_{0}^{2} / \chi^{4}+M^{2}}}=2 \pi \frac{\epsilon_{H}}{\sqrt{\epsilon_{H}^{2}+L_{0}^{2} / \chi^{2}}} .
$$

This result confirms that the specific energy loss is affected by the rotation to the second order of $(1 / \chi)$ for the case of high frequency and depends on thermal parameters $\epsilon_{T}$, 
finite thermal wave speed $c_{T}$ of G-N theory of thermoelasticity of type II, electromagnetic parameter $\epsilon_{H}$, and the transverse magnetic field $R_{H}$.

\section{REFERENCES}

[1] V. K. Agarwal, On surface waves in generalized thermoelasticity, J. Elasticity 8 (1978), 171177.

[2] M. A. Biot, Thermoelasticity and irreversible thermodynamics, J. Appl. Phys. 27 (1956), 240-253.

[3] P. Chadwick, Thermoelasticity. The dynamical theory, Progress in Solid Mechanics, Vol. 1, North-Holland Publishing, Amsterdam, 1960, pp. 263-328.

[4] D. S. Chandrasekharaiah, Thermoelastic plane waves without energy dissipation, Mech. Res. Comm. 23 (1996), no. 5, 549-555.

[5] - A uniqueness theorem in the theory of thermoelasticity without energy dissipation, J. Thermal Stresses 19 (1996), no. 3, 267-272.

[6] D. S. Chandrasekharaiah and K. S. Srinath, Axisymmetric thermoelastic interactions without energy dissipation in an unbounded body with cylindrical cavity, J. Elasticity 46 (1997), no. 1, 19-31.

[7] _ Thermoelastic plane waves without energy dissipation in a rotating body, Mech. Res. Comm. 24 (1997), no. 5, 551-560.

[8] _ Thermoelastic interactions without energy dissipation due to a point heat source, J. Elasticity 50 (1998), no. 2, 97-108.

[9] A. E. Green and P. M. Naghdi, Thermoelasticity without energy dissipation, J. Elasticity 31 (1993), no. 3, 189-208.

[10] H. Kolsky, Stress Waves in Solids, Monographs on the Physics and Chemistry of Materials, vol. 13, Clarendon Press, Oxford, 1953.

[11] H. W. Lord and Y. Shulman, A generalized dynamical theory of thermoelasticity, J. Mech. Phys. Solids 15 (1967), 299-309.

[12] A. Nayfeh and S. Nemat-Nasser, Thermoelastic waves in solids with thermal relaxation, Acta Mech. 12 (1971), 53-69.

[13] S. I. Pai, Some considerations of the fundamental equations of electro-magnetogasdynamics, Calcutta Math. Soc. Golden Jubilee Commemoration Vol. (1958/59), Part I, Calcutta Math. Soc., Calcutta, 1963, pp. 235-248.

[14] G. Paria, On magneto-thermo-elastic plane waves, Proc. Cambridge Philos. Soc. 58 (1962), 527-531.

[15] H. Parkus (ed.), Electromagnetic Interactions in Elastic Solids, CISM Courses and Lectures, vol. 257, Springer-Verlag, Vienna, 1979.

[16] P. Puri, Plane waves in generalized thermoelasticity, Internat. J. Engng. Sci. 11 (1973), $735-$ 744.

[17] S. K. Roychoudhuri, Electro-magneto-thermo-elastic plane waves in rotating media with thermal relaxation, Internat. J. Engng. Sci. 22 (1984), no. 5, 519-530.

[18] _ On magneto-thermo elastic plane waves in infinite rotating media with thermal relaxation, Electromagneto-Mechanical Interactions in Deformable Solids and Structures: Proceedings of the IUTAM Symposium (Tokyo, 1986) (Y. Yamamoto and K. Miya, eds.), Elsevier Science Publishers, Amsterdam, 1987, pp. 361-366.

[19] _ Magneto-thermo-micro elastic plane waves in finitely conducting solids with thermal relaxation, IUTAM Symposium on Mechanical Modeling of New Electromagnetic Materials (Stockholm, 1990) (R. K. T. Hsieh, ed.), Elsevier Science Publishers, Amsterdam, 1990, pp. 461-468.

[20] S. K. Roychoudhuri and L. Debnath, Magneto-thermo elastic plane waves in rotating media, Internat. J. Engng. Sci. 21 (1983), no. 2, 155-163.

[21] _ Magnetoelastic plane waves in infinite rotating media, J. Appl. Mech. 50 (1983), 283-287. 
[22] M. Schoenberg and D. Censor, Elastic waves in rotating media, Quart. Appl. Math. 31 (1973), 115-125.

[23] J. N. Sharma and R. S. Chauhan, On the problems of body forces and heat sources in thermoelasticity without energy dissipation, Indian J. Pure Appl. Math. 30 (1999), no. 6, 595-610.

[24] A. J. Wilson, The propagation of magneto-thermo elastic plane waves, Proc. Cambridge Philos. Soc. 59 (1963), 438-488.

S. K. Roychoudhuri: Department of Mathematics, Burdwan University, Bardhaman 713104, West Bengal, India

E-mail address: skrc_math@yahoo.com

Manidipa Banerjee (Chattopadhyay): Department of Mathematics, Burdwan University, Bardhaman 713104, West Bengal, India 


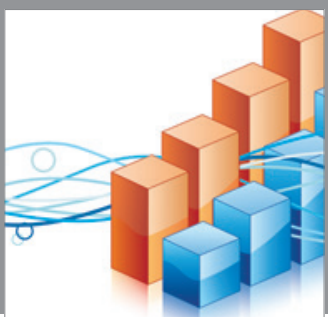

Advances in

Operations Research

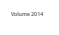

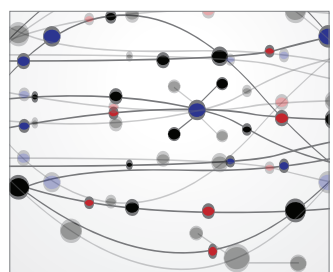

\section{The Scientific} World Journal
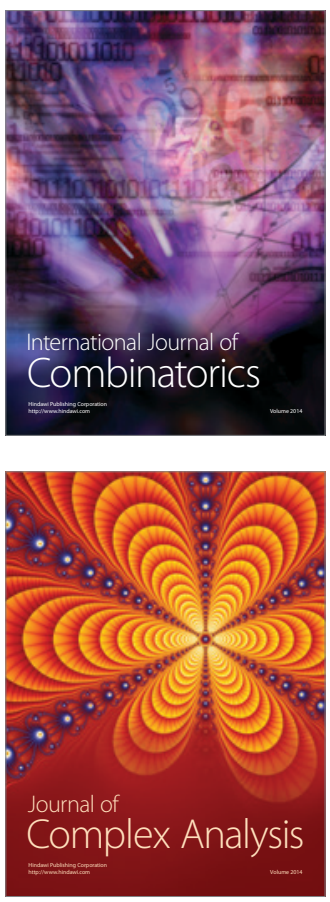

International Journal of

Mathematics and

Mathematical

Sciences
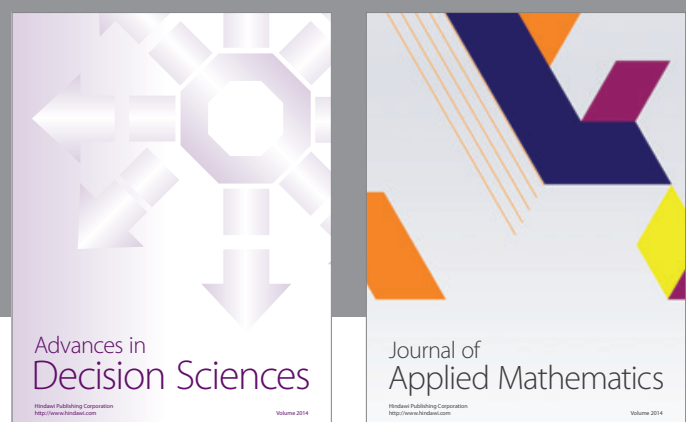

Journal of

Applied Mathematics
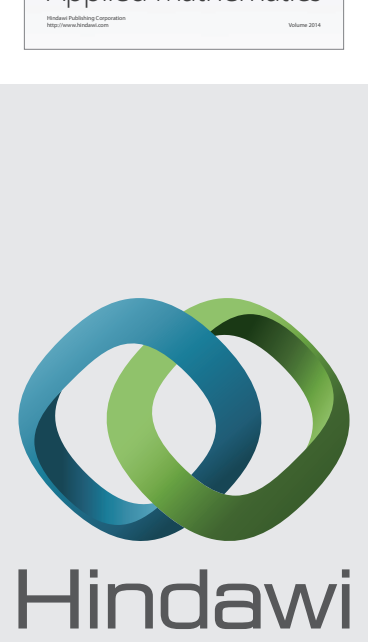

Submit your manuscripts at http://www.hindawi.com
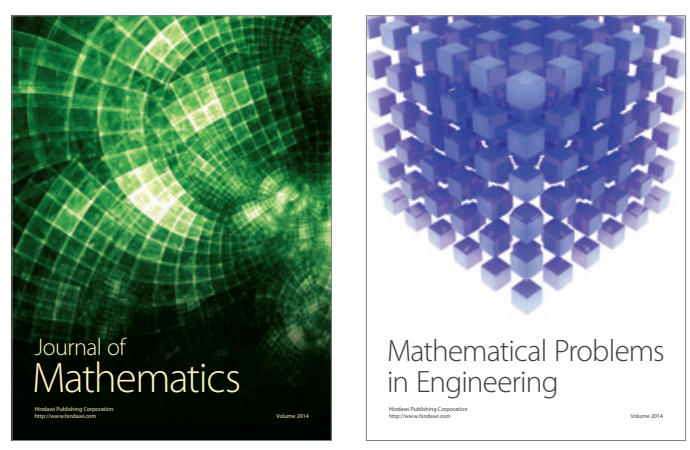

Mathematical Problems in Engineering
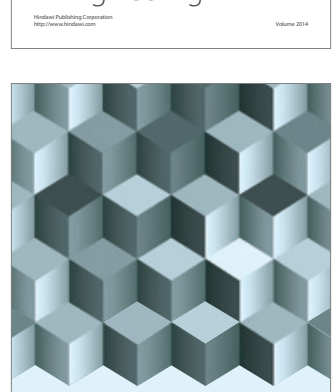

Journal of

Function Spaces
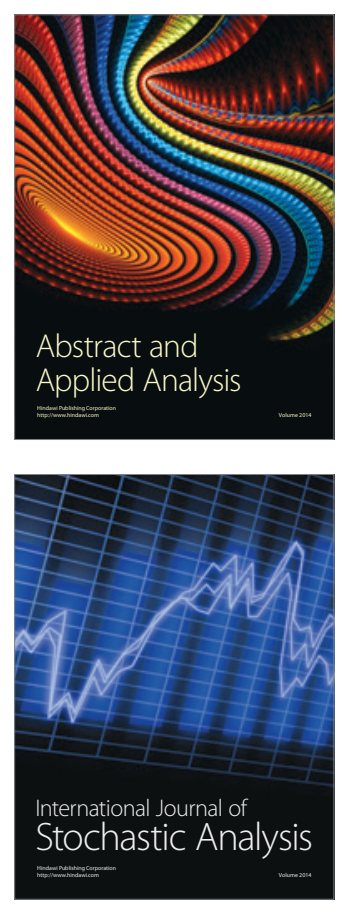

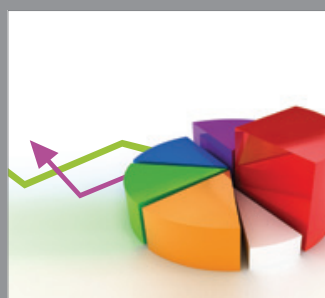

ournal of

Probability and Statistics

Promensencen
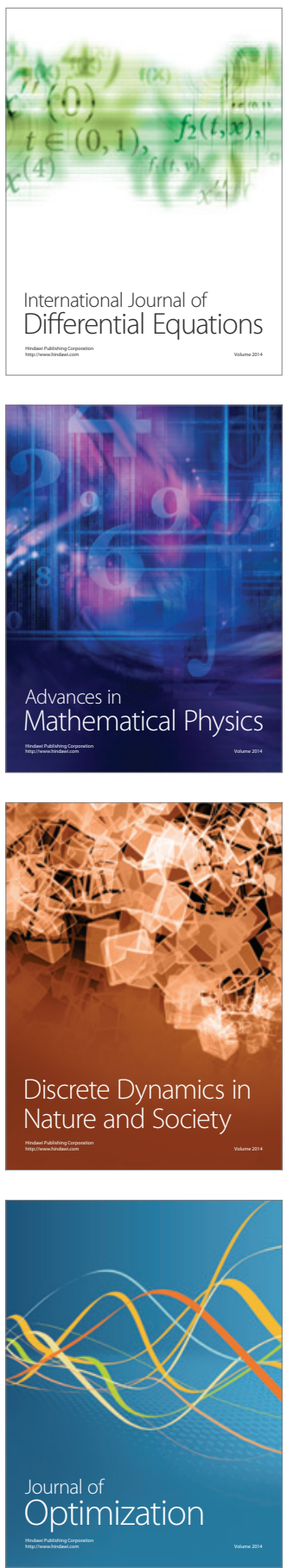